\title{
Epidemic Outbreak of Measles in the Region of Plovdiv, Bulgaria (March-July 2017)
}

\section{Petya Argirova',2, Andrei Petrov ${ }^{1,2}$, Nikolay Vatev ${ }^{3}$, Maria Atanasova ${ }^{4,5}$, Mariana Mourdjeva $^{4,5}$, Mariana Stoycheva ${ }^{1,2}$}

\author{
${ }^{1}$ Department of Infectious Diseases, Faculty of Medicine, Medical University, Plovdiv, Bulgaria \\ ${ }^{2}$ Clinic of Infectious Diseases, University Hospital “St. George”, Plovdiv, Bulgaria \\ ${ }^{3}$ Department of Epidemiology and Disaster Medicine, Faculty of Public Health, Medical University, Plovdiv, Bulgaria \\ ${ }^{4}$ Department of Microbiology and Clinical Immunology, Medical University, Plovdiv, Bulgaria \\ ${ }^{5}$ Immunology Section, Technological Center of Emergency Medicine, Plovdiv, Bulgaria \\ Email: nikolay_vatev@abv.bg
}

How to cite this paper: Argirova, P., Petrov, A., Vatev, N., Atanasova, M., Mourdjeva, M. and Stoycheva, M. (2018) Epidemic Outbreak of Measles in the Region of Plovdiv, Bulgaria (March-July 2017). World Journal of Vaccines, 8, 1-7.

https://doi.org/10.4236/wjv.2017.81001

Received: December 18, 2017

Accepted: February 2, 2018

Published: February 5, 2018

Copyright $\odot 2018$ by authors and Scientific Research Publishing Inc. This work is licensed under the Creative Commons Attribution International License (CC BY 4.0).

http://creativecommons.org/licenses/by/4.0/

\begin{abstract}
Object: To analyze the clinical, epidemiological and laboratory characteristics of the cases with measles in the Plovdiv region, Bulgaria, in the period March-July 2017. Materials and Methods: The study included 139 hospitalized patients with measles, treated in the Clinic of Infectious Diseases, University Hospital "St. George"-Plovdiv, during the observed period. Diagnosis was verified by ELISA in 133 of the patients. The following methods were used: clinical and epidemiological analysis, laboratory, microbiological and imaging tests. Results: The age distribution of the patients was: infants below 1 year were 23.8\%; 1 - 3 years-24.5\%; 4 - 17 years-30.9\%; and over 18 years $-20.8 \%$. Ethnicity: from Roma origin were $83.5 \%$ and Bulgarians- $16.5 \%$. Most of the patients (62\%) reported contact with a measles patient. Ninety eight of the patients were over 13 months of age and they should have been immunized, as the vaccine in Bulgaria is applied on the 13 month. Of these 98 patients, $49 \%$ were immunized, $28 \%$ were not, and for $23 \%$ there were not data. The most common clinical symptoms were: fever-97.1\%, cough-98.6\% and runny nose $-82.7 \%$. Conjunctivitis was observed in $82 \%$, asthenia in $46 \%$, and diarrhea in $51.1 \%$. The rash appeared average on the $4^{\text {th }}$ day of the onset of complaints. Koplik's spots were observed in $71.9 \%$, cervical lymphadenopathy in $56.1 \%$, hepatomegaly-in $25.2 \%$, and splenomegaly-in $7.9 \%$; auscultation data for bronchitis/pneumonia-in 33.1\%. Laboratory tests: leucopenia in $32.4 \%$, leukocytosis-2.2\%, normal results-in the rest $65.4 \%$. ESR was increased in $39.1 \%$ and CRP in $76.5 \%$ of the patients. X-ray of the lung was performed under indication and was positive in $28 / 46$. The serological test-anti-measles IgM (ELISA) was positive in $95.7 \%$ (133/139). We observed
\end{abstract}


the following complications: pneumonia-12.2\%, bronchitis- $15.1 \%$, laryngitis-4.3\%, otitis- $1.4 \%$, gastroenteritis- $14.4 \%$ and stomatitis-6.5\%. The outcome was fatal for one patient. Conclusions: Measles still prevails during the age of childhood, but $20.8 \%$ of the patients were adults. The analysis of the patient's immunity showed that the lap of immunization was the main cause for periodical arising of epidemic outbreaks, although the programs for elimination of measles in Europe until 2020. The disease ran with typical course but we observed a higher rate of complications.

\section{Keywords}

Measles, Epidemic Outbreak, Immunization, Complications

\section{Introduction}

Measles is a highly contagious vaccine-preventable viral infection, characterized by fever, cough, runny nose, conjunctivitis, enanthema (Koplik spots) and maculopapular rash [1]. The cause, Morbillivirus morbilorum, is RNA virus, which belongs to the Paramyxoviridae family, with a stable antigenic structure and identified 23 genotypes [2]. In the pre-immunization era, measles was a widespread disease from which nearly every child under 15 was ill. The disease is still common and even fatal in developing countries [3]. About 20 million people become diseased with measles worldwide, and 200,000 die annually, mainly children [1].

In 1963, a live attenuated measles vaccine was successfully licensed and started, and now the disease is rare, even unusual in most developed countries where the vaccine is widely used. However, measles continues to occur sporadically in the United States and major epidemics in developing countries make measles a constant cause for childhood morbidity and mortality. In 2000, measles was the fifth leading cause for child mortality worldwide, with nearly 777,000 deaths [4].

In 2002, the WHO American region was certified free of measles, but epidemic outbreaks have been reported in the European region, due to laps in the vaccination programs. In April 2009, after an absence of 7 years in Bulgaria an epidemic of measles was registered, after the virus spread from Hamburg, Germany. The disease quickly spread across the country, with a peak in March 2010, and declined at the end of the year. 24,253 patients were registered for a 2-year period in Bulgaria. These cases were $72 \%$ of those registered in Europe for 2010 [2].

According to ECDC, large epidemic outbreaks of measles in 2017 arose in Romania and Italy. Cases were reported despite intensified vaccination activities at national level. All countries in the EU/EEA reported cases of measles this year, with the exception of Latvia, Liechtenstein, Malta and Norway. In 2017, deaths were reported in Romania-21, Italy-3, Bulgaria-1, Germany-1, Portugal-1 
and France-1.

In 2017, by 30 August 2017, Germany reported 860 cases of measles, Ireland -8 , Italy -4328 cases, including 3 deaths; of all these cases in Italy 288 were among healthcare workers. The average age of the diseased was 27 years; $88 \%$ were not vaccinated and $7 \%$ have been received only one dose of the vaccine. In Romania, 6968 cases were reported in 2017. The risk of the spread and prolonged transmission of measles continues in areas with susceptible populations. Vaccination with at least two doses of MMR remains the most effective measure for the prevention of the disease [5].

In March 2017, an epidemic outbreak of measles occurred in the Plovdiv region, Bulgaria, which lasted until July 2017. The aim of this study is to analyze the clinical, epidemiological and laboratory characteristics of the measles patients in this outbreak.

\section{Materials and Methods}

The study included 139 measles patients treated in the Clinic of Infectious Diseases, University hospital "St. George"-Plovdiv in 2017. Inclusion criteria were laboratory confirmation of the cases with anti-measles IgM (ELISA) and/or presence of typical clinical manifestation and epidemiological link. Other patients in the clinic without characteristic clinical presentation of measles and lack of positive ELISA test for specific anti-measles antibodies were excluded from the study. The methods of clinical and epidemiological analysis were used; physical status; clinical-laboratory tests-complete blood count with differential count, ESR, biochemical parameters (CRP, aminotransferases) in some patients; microbiological studies-throat secretion, fecal samples (in case of indications); serological tests-proof of anti-Measles IgM, IgG with ELISA tests; X-ray of the lungs for some patients, if needed. ELISA tests were performed in the laboratory of the Dept. of Microbiology and Immunology, Medical University-Plovdiv, Bulgaria and in Technological Center of Emergency Medicine-Plovdiv, Immunology Section. The levels of anti-measles virus IgM antibodies were investigated in patients' sera using EUROIMMUN Anti-Measles Virus ELISA IgM qualitative kit with a cut-off ratio of 1.0. The ratio was calculated from the extinction values of the sample and the calibrators according to producer instructions. Serum samples were performed in duplicates using EUROIMMUN automatic ELISA analyzer. Anti-Measles Virus ELISA IgG were detected by quantitative ELISA EUROIMMUN kit. The results for anti-measles IgG were expressed in international units per litre (IU/l), with cut off $250 \mathrm{IU} / \mathrm{l}$. All positive serum samples for specific anti-measles antibodies were transported to the National Center of Infectious and Parasitic Diseases in Bulgaria where they were confirmed by PCR.

The study was approved from the ethical committee of Medical UniversityPlovdiv. Oral consent has been obtained from all of the patients before they have been included in the research. 


\section{Results}

Since 15 March 2017, when the first measles case was registered, 139 patients with measles were treated until July 2017, of whom 78 (56\%) men and 61 (44\%) women. The age distribution of the patients is presented in Table 1. The majority of the infected were children, the youngest patient was 2-months old, and the oldest one -57 years old.

The average hospital stay in the clinic was 6.4 days ( 3 - 15 days). According to the ethnic group, the Roma predominated-116 (83.5\%), and the Bulgarians were $23(16.5 \%) .86(62 \%)$ of the patients reported a contact with another measles patient.

The immunization status of the patients was as follows: 98 of them were older than 13 months, and should have been immunized with MMR, as in Bulgaria the vaccine is applied routinely at the 13th month. Only 48 (49\%) said that they had been immunized, 27 (28\%) were not immunized, and for 23 (23\%) of them there were no data. The serological tests conducted in the group of breastfed infants showed that only 2 had anti-measles IgG. From the remaining children and adults who should have been immunized (a total of 106), 55 (52\%) were antimeasles IgG negative.

Some of the patients had the following additional diseases: anemia-2, epilepsy -1 , hydrocephalus -1 , recurrent pneumonia-2, frequent febrile convulsions -1 , diabetes mellitus -1 , hyperthyroidism -1 , past lung tuberculosis -1 , hepatitis $\mathrm{C}-1$ and arterial hypertension -2 . This had no influence on the course of measles. The clinical symptoms which we observed are presented in Table 2.

Laboratory and imaging results:

- 45 (32.4\%) of the patients had leucopenia, 3 (2.2\%) had leukocytosis, and the rest-normal levels.

- ESR was elevated for 54 (39.1\%) of the patients-the highest value was 59 $\mathrm{mm} / \mathrm{h}$ (normal $-<20$ for adults and $<12$ for children).

- CRP for the 89 studied patients was elevated in 68 (76.5\%) of them-the highest value was $67 \mathrm{mg} / \mathrm{l}$ (normal level $-<8 \mathrm{mg} / \mathrm{l}$ ).

- ALT was elevated for half of the patients.

- Throat secretion was studied in all patients. Str. pneumoniae was isolated in only one child, the others were negative.

- Fecal samples were studied in patients with diarrhea, but pathogenic microorganisms were not isolated from anyone.

Table 1. Age distribution of the patients.

\begin{tabular}{ccc}
\hline Age & Number of patients & Percent \\
\hline$<1 \mathrm{y}$. & 33 & $23.8 \%$ \\
$1-3 \mathrm{y}$. & 34 & $24.5 \%$ \\
$4-17 \mathrm{y}$. & 43 & $30.9 \%$ \\
$>18 \mathrm{y}$. & 29 & $20.8 \%$ \\
\hline
\end{tabular}


Table 2. Frequency of underlying clinical symptoms.

\begin{tabular}{ccc}
\hline Symptoms & Number of diseased & Percentage \\
\hline Fever & 135 & $97.1 \%$ \\
Cough & 137 & $\mathbf{9 8 . 6 \%}$ \\
Runny nose & 115 & $\mathbf{8 2 . 7 \%}$ \\
Asthenia or adynamia & 64 & $46 \%$ \\
Diarrhea & 71 & $51.1 \%$ \\
Sore throat & 17 & $12.2 \%$ \\
Vomiting & 34 & $24.5 \%$ \\
Abdominal pain & 14 & 10.0 \\
Epistaxis & 5 & $3.6 \%$ \\
Objective symptoms & & \\
Rash & 139 & $\mathbf{1 0 0 \%}$ \\
Conjunctivitis & 114 & $\mathbf{8 2 . 1 \%}$ \\
Koplik spots & 100 & $71.9 \%$ \\
Pulmonary finds (dry and wet wheezing) & 78 & $56.1 \%$ \\
Splenomegaly & 35 & $25.2 \%$ \\
Hepatomegaly & 11 & $7.9 \%$ \\
\hline
\end{tabular}

${ }^{*}$ The rash occurred average on the $4^{\text {th }}$ day of the onset of complaints ( $1-7$ days), and in most cases the typical 3-day stage of rash, localization and spread was observed, type-maculopapular, more often confluent. In few cases, there was insignificant amount of rash, which was atypical and rapid transient. In two cases, the rash was hemorrhagic.

- Lung X-ray was performed for 46 and was positive (X-ray of pneumonia or bronchitis) for 28 (61\%) and negative for 18 (39\%) of them.

- The serological test for anti-Measles IgM was positive in 133 (95.7\%), for 2 in the gray area, and 4 patients were negative, but with typical clinical symptoms for the disease.

In the course of the disease, many complications affecting the respiratory tract and organs of the digestive system were observed (Table 3). Neurological complications were not observed.

Of our patients, 138 recovered, and 1 died. The infected child that died was a 9-month old infant with severe laryngitis, progressive worsening of the condition, complicated with laryngospasm and acute respiratory failure.

\section{Discussion}

To be achieved the measles elimination it is required monitoring of the susceptibility of the population to the disease in order to prevent outbreaks in the future [6].

The age distribution of our patients showed that the majority of the diseased were children, whereas there were and adults-20\%. In the same time, a study from Slovakia showed that people aged 30 - 45 and non-vaccinated children 
Table 3. Observed complications in patients with measles.

\begin{tabular}{ccc}
\hline Complications affecting the respiratory system & Number of diseased & Percentage \\
\hline Bronchitis & 21 & $15.1 \%$ \\
Pneumonia & 17 & $12.2 \%$ \\
Laryngitis & 6 & $4.3 \%$ \\
Otitis & 2 & $1.4 \%$ \\
\hline Complications affecting the digestive system & & \\
\hline Gastroenteritis & 20 & $14.4 \%$ \\
Stomatitis & 9 & $6.5 \%$ \\
Hepatitis & 4 & $2.9 \%$ \\
Total & 79 & $57 \%$ \\
\hline
\end{tabular}

were the most affected age groups [6]. In our patients, measles occurred typically, with disease-specific symptoms and laboratory changes, but with a very high incidence of complications (57\%), unlike those in Belgium, where an atypical clinical presentation was observed, but also severe complications, most often dehydration, pneumonia, hepatitis, gastrointestinal problems, otitis, isolated cases of febrile seizure, encephalitis, pancreatitis and uveitis. No deaths were reported there [7], whereas one child died in our study. Of our patients, $28 \%$ older than 13 months, were not immunized against measles, similar to data from other authors [7]; however, in some studies a much higher percentage of non-immunized patients was observed-88\% [9].

In Italy the immunization coverage against measles was insufficient (it varied between $85.4 \%$ and $90.6 \%$ in the period $2007-2016$ ), and there are large groups of susceptible adults [8]. The authors described the ongoing epidemic of measles for the period January to August 2017, with over 4400 cases. The highest incidence was in infants below 1 year, whereas $7 \%$ occurred among healthcare workers; 3 fatal cases and 2 with encephalitis were reported. Complications were reported in $35.1 \%$ of the cases: diarrhea (16\%), stomatitis (13.9\%), keratoconjunctivitis (9.3\%), hepatitis (8.7\%), respiratory failure (6.3\%), otitis (4.6\%), thrombocytopenia (3.2\%) [9]. No patients among contact healthcare workers were observed in Bulgaria.

Limitations about the study: Unfortunately we could not obtain access to the documents for registration of the immunizations for all of the patients. That's why for some of them there were not data for their immunization status.

This epidemic outbreak of measles in the Plovdiv region, Bulgaria, was significantly smaller than the one in 2010-then in the same region more than 2, 800 people were diseased, mainly children [10]. However, in this outbreak significant laps in the immunization coverage were also observed.

\section{Conclusion}

Measles still prevails during the age of childhood, but $20.8 \%$ of the patients were 
adults. The analysis of the patient's immunity showed that the lap of immunization was the main cause for periodical arising of epidemic outbreaks, despite the programs for elimination of measles in Europe until 2020. The disease ran with typical course but we observed a higher rate of the complications.

\section{References}

[1] Poster, R. and Kaplan, J. (2011) The Merck Manual of Diagnosis and Therapy. Merck Publishing Group, Rahway, 1458-1460.

[2] Argirova, R. and Komitova, R. (2012) About the Vaccines. Monograph. In: Argirova, R., Ed., Measles-A Record Progress, but with High Risk of Reemerging. "V. Petrov", Plovdiv, 116-125. (In Bulgarian)

[3] Centers for Disease Control and Prevention (2015) Epidemiology and Prevention of Vaccine-Preventable Diseases. 13th Edition, Merck Publishing Group, Rahway, 209-299.

[4] Gershon, A. (2010) Harrison's Infectious Diseases. 17th Edition, Merck Publishing Group, Rahway, 947-951.

[5] European Center for Disease Prevention and Control (2017) Epidemiological Update: Measles-Monitoring European Outbreaks.

[6] Zibolenová, J., Chladná, Z., Švihrová, V., Baška, T., Waczulíková, I. and Hudečková, H. (2017) Estimation of the Population Suspectibility against Measles in Slovakia. Central European Journal of Public Health, 25, 46-54.

https://doi.org/10.21101/cejph.a4914

[7] Grammens, T., Schirvel, C., Leenen, S., Shodu, N., Hutse, V., Mendes da Costa, E. and Sabbe, M. (2017) Ongoing Measles Outbreak in Wallonia, Belgium, December 2016 to March 2017: Characteristics and Challenges. Euro Surveillance, 22, pii=30524. https://doi.org/10.2807/1560-7917.ES.2017.22.17.30524

[8] Porretta, A., Quattrone, F., Aquino, F., Pieve, G., Bruni, B., Gemignani, G., Vatteroni, M.L., Pistello, M., Privitera, G.P. and Lopalco, P.L. (2017) A Nosocomial Measles Outbreak in Italy, February-April 2017. Euro Surveillance, 22, pii=30597.

https://doi.org/10.2807/1560-7917.ES.2017.22.33.30597

[9] Filia, A., Bella, A., Del Manso, M., Baggieri, M., Magurano, F. and Rota, M.C. (2017) Ongoing Outbreak with Well over 4,000 Measles Cases in Italy from January to End August 2017-What Is Making Elimination So Difficult? Euro Surveillance, 22, pii=30614. https://doi.org/10.2807/1560-7917.ES.2017.22.37.30614

[10] Vatev, N., Stoycheva, M., Petrov, A. and Velcheva, R. (2012) Epidemic Outbreaks in Plovdiv Region (Bulgaria) of Vaccine-Preventable Diseases: Measles, Mumps, Hepatitis A (2006-2010). World Journal of Vaccines, 2, 172-177. https://doi.org/10.4236/wjv.2012.23023 